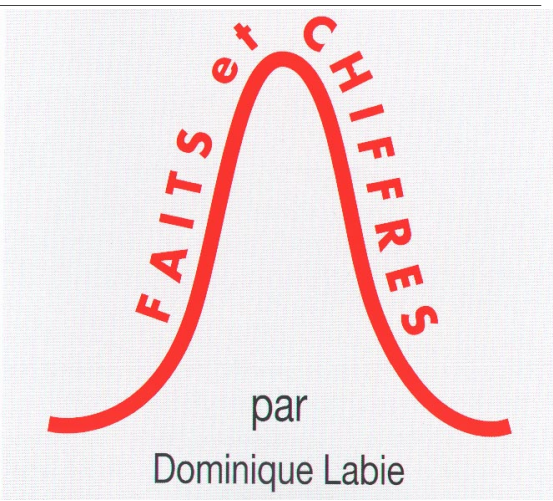

médecine/sciences $1999 ; 15$ : 1064-6

\title{
Les paradoxes de la transition démographique au Brésil
}

$\mathbf{L}$ a transition démographique, avec baisse de la fécondité, est un paramètre habituel du développement, qui traduit le changement d'attitude des nouvelles générations sur la taille de la famille désirée. Déjà ancienne dans nos pays industrialisés, elle a touché progressivement d'autres continents. Les facteurs invoqués pour en expliquer le caractère progressif sont, avec l'industrialisation, la diffusion de l'instruction parmi les femmes, accélérée souvent par une action publique forte en matière de planification familiale. Elle a été un phénomène relativement récent au Brésil, amorcé au milieu des années 1960, mais par ailleurs exceptionnellement rapide, puisqu'en trois décennies l'indice synthétique de fécondité est tombé de 5,7 à 2,3 enfants par femme et semble en passe d'atteindre sa phase finale. Cette vitesse et ce calendrier sont comparables aux données relevées au Mexique, alors que dans les pays du cône sud, l'Argentine par exemple, la même évolution s'étale sur tout le $\mathrm{XX}^{\mathrm{e}}$ siècle. Les causes invoquées plus haut ne semblent pas suffisantes pour expliquer ce paradoxe, et une étude récente de l'Institut national d'études démographiques (INED) apporte là-dessus des données complémentaires [1].

Le Brésil est un pays-continent, vaste comme seize fois la France et plus de deux fois l'Inde, l'un des plus grands pays du monde. Son évolution démographique a été exceptionnellement rapide, puisqu'au moment de l'indépendance, en 1822, il ne comptait repères ensuite donnent 18 millions en 1900, 54 millions en 1950, et 147 millions au recensement de 1991. Dans cet accroissement de population, il faut faire intervenir, comme aux États-Unis, une immigration européenne (les principaux contingents étant en ordre décroissant les Italiens, les Portugais, les Espagnols et les Allemands), mais aussi la traite des esclaves. On estime qu'en trois siècles le Brésil a reçu entre 3 et 5 millions d'Africains; l'interdiction de la traite date de 1850, l'abolition de l'esclavage seulement de 1888. A cette évolution globale de la population, il faut ajouter une évolution du taux d'urbanisation, lui aussi très rapide, un des plus élevés du monde : 4 Brésiliens sur 5 vivent actuellement en ville, alors que les deux tiers vivaient en zone rurale en 1950. Ces dernières décennies, comme ailleurs, s'accompagnent d'une baisse des taux de mortalité, de $20,9 \%$ à $14,2 \%$, et d'une baisse des taux bruts de natalité. L'ensemble de ces données s'est traduit par un accroissement maximal de la population aux environs de 1960.

Le Brésil est aussi un pays extrêmement hétérogène. L'hétérogénéité est d'abord géographique et climatique: le Brésil s'étend sur près de 30 degrés de latitude, et traverse d'est en ouest presque la totalité de l'Amérique du Sud. Elle est aussi une hétérogénéité de peuplement. Outre le district fédéral, le Brésil comprend 26 états que l'on peut regrouper en cinq régions géographiques que tout distingue et qui sont en fait des pays différents (figure 1). La région Nord comporte l'Amazonie, la région la plus étendue, mais aussi la moins peuplée, moins de 3 habitants au kilomètre carré. Le Centre-Ouest, peu peuplé également, est cependant en forte croissance. Le Sud, Rio Grande do Sul, voisin de l'Argentine et de l'Uruguay, s'en rapproche par un peuplement européen dont la densité est supérieure à 40 habitants. Le cœur industriel du pays reste cependant le Sudeste, où sont concentrées les plus grandes villes du pays. Quant au Nordeste, berceau du Brésil colonial, c'est un pays pauvre, au climat hostile, dont la population décroît par émigration. La même hétérogénéité se retrouve au niveau du produit par habitant, voisin de ceux de l'Argentine ou du Chili dans le Sud, mais proches ailleurs de ce qu'on observe dans des pays pauvres comme le Paraguay. L'espérance de vie, les taux de mortalité infantile enfin, sont aussi le reflet de conditions sociales et sanitaires variables, ainsi que l'analphabétisme chez les adultes $(10 \%$ dans le Sud, $27 \%$ dans le Nordeste). Dans un pays où le développement social a peu été étayé par la politique des pouvoirs publics, l'hétérogénéité se retrouve aussi dans le dualisme du développement, même celui des régions riches. Le processus d'urbanisation accéléré y a produit simultanément des quartiers urbains modernes comparables à ceux des pays développés et des favelas dépourvues des services de base, et dont les problèmes sanitaires sont encore ceux d'un autre âge.

$m / s n^{\circ} 8-9$, vol. 15, août-septembre 99 


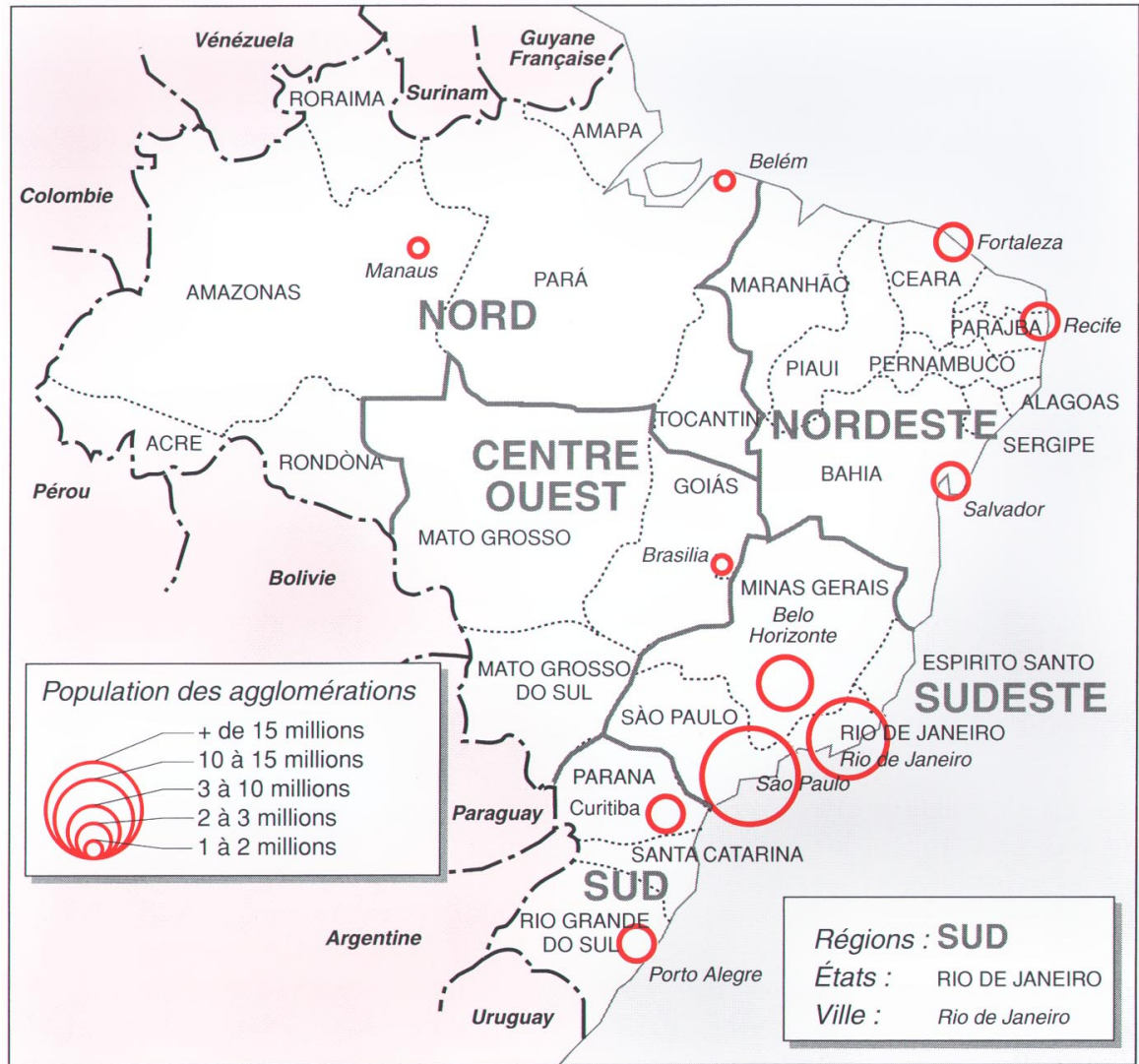

Figure 1. Répartition du Brésil en cinq grandes régions. Les agglomérations urbaines dont la population dépasse un million d'habitants sont indiquées par des cercles rouges de tailles croissantes. On voit que la concentration urbaine est majoritaire dans le Sudeste.

Contrastant avec cette hétérogénéité géographique, sociale et économique, le paradoxe est ici la relative homogénéité du rythme dans la baisse de fécondité. Amorcée vers 1960, cette transition vers une fécondité faible s'accélère vers 1970 , et concerne toutes les régions, s'opérant de façon d'autant plus rapide que le taux initial était plus élevé (Tableau I). La planification familiale, inscrite en 1983 dans un Programme d'assistance intégrale à la santé de la femme, n'a pourtant été appliquée que de façon très inégale, et n'a été encouragée que par des organisations non gouvernementales et des organismes privés. L'explication est à rechercher ailleurs, dans un taux élevé d'utilisation de la contraception. Une enquête nationale en 1986 montrait qu'elle touchait $70 \%$ des femmes, avec des proportions sensiblement équivalentes de stérilisation et d'utilisation de la pilule; dix ans plus tard, en 1996, la méthode de la stéri-
Peut-on remonter plus loin encore dans l'explication? La stérilisation représente, évidemment une solution de facilité, peu coûteuse par ailleurs, et qui ne demande pas, comme la contraception orale, d'adaptation culturelle. Elle est devenue la solution pratique, coïncidant la plupart du temps avec le dernier accouchement souhaité. A l'appui de cette pratique, il faut noter une autre spécificité du Brésil : la fréquence des césariennes y est une des plus élevées du monde, $36 \%$ des naissances ces dernières années. Considérée comme un confort moderne, la césarienne semble intégrée à la culture féminine comme à la culture médicale. Elle permet, à la demande ou sur recommandation, une ligature des trompes considérée comme souhaitable. Là encore, on retrouve un élément d'hétérogénéité, et le calendrier de fécondité subit une polarisation sociale: alors que l'âge de la mère lors de la première naissance est devenu plus tardif dans les milieux riches et/ou instruits, il reste précoce chez les pauvres, les grossesses d'adolescentes y étant fréquentes. En milieu populaire, également, l'âge de la stérilisation a tendance à baisser, inférieur à 30 ans, en moyenne. Plus de $20 \%$ des femmes ont été stérilisées avant 25 ans. La pyramide des âges se ressent déjà, au Brésil, de cette chute rapide de la fécondité, et les enfants jeunes y sont aujourd'hui moins nombreux que les adolescents. Les projections démographiques sont actuellement révisées à la baisse.

C'est donc par la conjonction de deux séries de paramètres qu'on pourrait expliquer les données apparemment paradoxales de la transition démographique au Brésil. Il y
Tableau I

TAUX SYNTHÉTIQUES DE FÉCONDITÉ AU BRÉSIL ET DANS LES GRANDES RÉGIONS (1975-1996) (NOMBRE D'ENFANTS PAR FEMME)

\begin{tabular}{|lccccccc|}
\hline & 1965 & 1970 & 1975 & 1980 & 1985 & 1990 & 1996 \\
\hline Brésil & 5,7 & 5,4 & 4,8 & 4,0 & 3,3 & 2,7 & 2,3 \\
Nord & 7,0 & 6,7 & 6,2 & 5,5 & 4,8 & 4,0 & 2,8 \\
Nordeste & 7,2 & 6,9 & 6,4 & 5,8 & 4,9 & 4,0 & 2,9 \\
Centre-Ouest & 6,4 & 5,9 & 5,1 & 4,2 & 3,4 & 2,9 & 2,3 \\
Sud & 5,7 & 5,2 & 4,4 & 3,4 & 2,7 & 2,3 & 2,1 \\
Sudeste & 4,7 & 4,4 & 3,8 & 3,2 & 2,7 & 2,4 & 2,0 \\
\hline
\end{tabular}


aurait en premier lieu un taux et une rapidité d'urbanisation parmi les plus élevés du monde, qu'accompagnent de fortes migrations interrégionales. Les conditions de la vie urbaine poussent toujours à la réduction de la taille des familles. A ce phénomène purement démographique s'est ajouté un mode de culture de la reproduction, qui touche les femmes mais aussi le milieu médical, et qui inclut l'avortement, la contraception orale, mais surtout, de façon très spécifique, la césarien- ne souvent accompagnée de stérilisation. L'excès vient d'en être reconnu et une campagne est tentée par le ministère de la Santé, dont il n'est pas sûr qu'elle puisse enrayer des pratiques très entrées dans les mours

\section{RÉFÉRENCES}

1. Bozon M, Enoch E. Brésil : la transition démographique rapide d'un pays hétérogène. Population et Sociétés $1999 ; n^{3} 345$.

\section{Dominique Labie}

Inserm U. 129, ICGM, CHU Cochin, 24, rue du Faubourg-Saint-Jacques, 75014 Paris, France.

\section{口 BRÈVES}

- $\quad$ Sur les traces des dinosaures. Depuis le succès du roman Jurassic Park*, l'engouement pour ces grands disparus, les dinosaures, ne s'est pas départi. Et si l'étude de leur $\mathrm{ADN}$ a pu laisser sceptique $\left(\mathrm{m} / \mathrm{s}\right.$ 1995, $n^{\circ} 4$, p. 631), celle des empreintes de leur pattes, qui vient de nous parvenir du Groenland, de l'époque du trias tardif (> 200 millions d'années), ne saurait nous laisser indifférents. Car l'ichnologie - la science des empreintes - est plus qu'une science purement anatomique. Elle fournit de précieux renseignements sur la locomotion (posture, démarche et vitesse), à condition, bien sûr, de connaître la consistance du sol dans lequel les empreintes furent laissées. De nombreux livres ont été écrits par les ichnologues, sur toutes sortes d'espèces animales vivantes ou disparues, et en particulier sur les traces des dinosaures, retrouvées un peu partout, en Afrique, en Chine, dans le Nouveau Monde (Argentine; Connecticut, Virginie et Texas, USA) [1-5]. Mais les empreintes de la région Est du Groenland (Jameson Land), où de très nombreux fossiles furent retrouvés, sont particulièrement instructives. Elles ont été faites dans des couches sédimentaires de sable constitué de petits grains arrondis de carbonate et de silice, et laissées là au cours de la formation du Fjord Fleming. Elles proviennent de théropodes, un sous-ordre de dinosaures carnas- siers qui comprend deux superfamilles : les cælusauriens et les carnosauriens (parmi lesquels on trouve le redoutable tyrannosaure). Des chercheurs américains, spécialistes des dinosaures, viennent de faire une étude soigneuse de ces empreintes en tenant compte de nombreux paramètres : la profondeur des pas, la nature, la pente et la consistance du terrain, avec reconstitution en coupes [6]. Ils ont ensuite effectué des études comparatives avec des empreintes de pattes de pintade et de dinde. Puis ils ont reconstitué, en images informatisées, les déplacements dans le sol meuble au fur et à mesure que les pattes s'enfoncent, doigts écartés, puis se retirent, doigts resserrés, avec un mouvement en dorsiflexion. Ce mouvement est identique à celui des oiseaux actuels. Trois doigts sont visibles; le cinquième est absent ; l'hallux (premier doigt) est plus court, appliqué verticalement contre le deuxième métatarsien. Il n'est certainement pas retourné vers l'arrière (pour former une pince comme chez les oiseaux capables de se percher sur des branches). Ces dinosaures théropodes étaient donc adaptés à la marche et à la course mais ils n'étaient pas plantigrades (leur talon était toutefois un peu plus bas que celui des oiseaux à vol restreint ou nul d'aujourd'hui, mais sans que la plante ne touche com- plètement le sol dans sa surface tarso-métatarsienne). Enfin, le déplacement des pattes postérieures impliquait beaucoup plus la mobilisation des fémurs, comme chez les crocodiles. Ainsi, on le voit, l'ichnologie, et les dinosaures, ont encore un bel avenir devant eux.

[1. Gillette DD, Lockey MG. Dinosaur tracks and traces. New York: Cambridge University Press, 1989.]

[2. Thulborn T. Dinosaur tracks. London : Chapman Hall, 1990.]

[3. Murie OJA. Field guide to animal tracks. Boston : Hougton Mifflin, 1968.]

[4. Matsukawa M, et al. Palaios $1995 ; 10: 3-15$.

[5. Weems RE, Kimmel PG. P Biol

Soc Wash 1993 ; 106 : 390-401.]

[6. Gatesy SM, et al. Nature 1999 ; 399 : 141-4.]

\footnotetext{
* Jurassic Park. Michael Crichton, Paris : Robert Laffont, 1992.
} 